\title{
ASPECTOS EPIDEMIOLÓGICOS DE HEPATITE B E C NO MUNICÍPIO DE CASCAVEL/PR NO PERÍODO DE 2013 A 2017
}

\section{EPIDEMIOLOGICAL ASPECTS OF HEPATITIS B AND C IN THE CITY OF CASCAVEL/PR FROM 2013 TO 2017}

\author{
Ana Beatriz de Araujo Berton ${ }^{1 *}$, Luciana Osório Cavalli² \\ ${ }^{1}$ Acadêmica, de Medicina do Centro Universitário FAG. \\ 2 Médica, mestre em Biociências e Saúde, Docente do curso de Medicina do Centro Universitário \\ FAG. \\ * Autor correspondente: biaaberton@hotmail.com https://orcid.org/0000-0002-8350-1438
}

DOI: $10.35984 / f j h . v 2 i 1.157$

\begin{abstract}
RESUMO
As hepatites $B$ e $C$ são doenças que afetam o fígado e podem acarretar graves problemas se não diagnosticadas e tratadas de maneira correta e precocemente. Este estudo visa analisar o sexo, a faixa etária, a provável fonte de contaminação, a forma clínica em que os pacientes foram diagnosticados e a situação vacinal em que os mesmos se encontravam, no caso da hepatite B, notificadas no Departamento de Vigilância Epidemiológica da Secretaria de Saúde do Município de Cascavel/PR no período de 2013 a 2017, para que medidas sejam tomadas a fim de que haja redução dos casos dessas doenças no município e região, já que o oeste do Paraná tem alta taxa de prevalência das mesmas. Esse estudo demonstrou que nessa região o sexo afetado predominantemente foi o masculino e a provável fonte de contaminação foi a sexual, nos dois tipos de hepatite estudados.
\end{abstract}

Palavras-chave: Hepatite B. Hepatite C. Dados epidemiológicos. Cascavel/PR.

\begin{abstract}
Hepatitis $B$ and $C$ are diseases that affect the liver and can cause huge problems if not diagnosed and treated properly and early. This study aims to analyze the gender, age, probable source of contamination, the clinical form in which the patients were diagnosed and the vaccination situation in which they were, in the case of hepatitis $B$, notified in the Department of Epidemiological Surveillance that belongs to the Department of Health of Cascavel, in the state of Paraná, in the period from 2013 to 2017, to take measures to reduce cases of such diseases in the city and region, since the west of the Paraná has high prevalence rate of these diseases. This study demonstrated that in this region the predominantly affected gender was male and the probable source of contamination was through sexual intercourse in the two types of hepatitis studied.
\end{abstract}

Keywords: Hepatitis B. Hepatitis C. Epidemiological data. Cascavel/PR.

\section{INTRODUÇÃO}

De acordo com o Ministério da Saúde (2008) "as hepatites virais são doenças provocadas por diferentes agentes etiológicos, com tropismo primário pelo tecido hepático, que apresentam características epidemiológicas, clínicas e laboratoriais semelhantes, porém com importantes particularidades".

A hepatite viral é a mais importante causa de doenças hepáticas e as hepatites

B e C possuem potencial de evoluir para hepatite crônica, cirrose e carcinoma 
hepatocelular, desse modo aumentando a mortalidade de doenças crônicas do fígado e a incidência de carcinoma hepatocelular relacionada (SHEPARD, 2006, apud XIMENES et al, 2010).

"O Estado do Paraná vem se despontando em notificações de hepatite B na região Sul do País com 14,4\%, atrás apenas do Estado de Santa Catarina (19,7\%) em relação às notificações em nível nacional" são dados obtidos pela Secretaria de Vigilância em Saúde, Ministério da Saúde (BORTOLUCCI, FERREIRA E CORREA, 2015). Segundo Bortolucci, Ferreira e Correa (2015) as cinco cidades que mais tiveram casos de hepatite B no Paraná entre 2008 a 2013 foram Curitiba $(17,98 \%)$, Foz do Iguaçu (13,13\%), Cascavel (13,06\%), Toledo (8,02\%) e Londrina $(6,73 \%$ entre 2008 e 2009).

"Desde 2000, a vacina contra hepatite B é obrigatória para todos os recémnascidos e indivíduos com idade até 49 anos, para garantir a proteção imunológica por pelo menos 20 anos. No entanto, apesar da vacinação obrigatória, existem várias barreiras para alcançar a vacinação de toda a população alvo, incluindo medos de reações adversas, ausência de informações sobre a transmissão do vírus e risco de infecção, falta de tempo de trabalho e dificuldade de acesso à imunização" (ASSUNÇÃO, 2012, apud VIEIRA et al, 2015, p. 18-21, tradução nossa).

Esse trabalho visa dimensionar a epidemiologia de hepatite $\mathrm{B}$ e $\mathrm{C}$ na região de Cascavel, já que diversos estudos sugerem que é a região com maior prevalência desta doença no Paraná. Com este estudo será possível estabelecer futuras estratégias para prevenção dessas doenças e assim reduzir a taxa de incidência nessa região. Permitirá também identificar a faixa etária e sexo mais comuns de ocorrer hepatite $B$ e a hepatite $C$, identifica a situação vacinal para hepatite $B$ nos casos notificados, verifica a provável fonte de contaminação dos casos notificados e identifica em qual forma clínica a hepatite $B$ ou hepatite $C$ foram diagnosticadas.

\section{METODOLOGIA}

Esse trabalho foi feito com finalidade básica, em tempo longitudinal e retrospectivo, de natureza observacional e a forma de abordagem utilizada é qualitativa e quantitativa, sendo a última analítica e descritiva. Tem objetivo exploratório e foi feito a partir de pesquisa bibliográfica e documental.

O referencial teórico deste estudo também é composto por artigos disponíveis em base de dados online. Foi necessário a aprovação deste estudo pelo Comitê de Ética em Pesquisa com Seres Humanos já que foram utilizados dados epidemiológicos de domínio público e aprovado sob CAAE 06637118.9.0000.5219.

\section{RESULTADOS E DISCUSSÃO}

A amostra disponibilizada pelo Departamento de Vigilância Epidemiológica da Secretaria de Saúde do Município de Cascavel/PR foi de 1.387 casos notificados no período de 2013 a 2017 no município, sendo 975 casos de hepatite B e 412 casos de hepatite $\mathrm{C}$.

Os resultados demonstraram que o sexo predominantemente atingido na hepatite $B$ foi o masculino com 553 casos notificados (56,3\%), enquanto o feminino teve 422 casos. Na hepatite $\mathrm{C}$ também predominou o número de casos no sexo masculino, com 228 casos correspondendo 55,33\%, já o feminino com 184 casos. Em 
contrapartida, Gonçalves et al. (2019) afirma que "o maior percentual de casos de hepatite $\mathrm{B} \mathrm{e} \mathrm{C}$, ao longo da série estudada, ocorreu em indivíduos do gênero feminino, com 53,81\%, na idade adulta (19 a 59 anos), com $77,99 \%$, na etnia parda, com $77,95 \%$, e ensino fundamental ( $5^{\mathrm{a}}$ a $8^{\mathrm{a}}$ série), com $35,04 \% "$.

$\mathrm{O}$ ano de 2014 foi o que teve maior número de casos notificados de hepatite $\mathrm{B}$ no período estudado, aproximadamente $27 \%$, sendo 161 homens e 108 mulheres. Com relação à hepatite C, o ano com maior número de notificações foi o de 2015 $(24,5 \%)$, porém diferentemente da hepatite C, o predomínio foi no sexo feminino, sendo 58 casos notificados em mulheres e 43 em homens.

Quanto à faixa etária na hepatite $\mathrm{B}$, no sexo masculino houve predominância de 148 notificações dos 35 aos 44 anos, enquanto a que teve menor número de notificações foi dos 5 aos 14 anos, com apenas 2 casos. No sexo feminino predominou a faixa etária dos 25 aos 34 anos, com 101 notificações. Como no sexo masculino, o menor número de casos foi na faixa etária dos 5 aos 14 anos, com apenas 1 caso, entretanto também houve 1 notificação nos menores de 1 ano de idade.

Tabela 1. Casos notificados por sexo e faixa etário da hepatite B.

\begin{tabular}{ccc}
\hline Faixa etária & $\begin{array}{c}\text { Casos notificados no sexo } \\
\text { masculino }\end{array}$ & $\begin{array}{c}\text { Casos notificados no sexo } \\
\text { feminino }\end{array}$ \\
\hline$<1$ & 0 & 1 \\
$\mathbf{5}-14$ & 2 & 1 \\
$\mathbf{1 5 - 2 4}$ & 43 & 55 \\
$\mathbf{2 5 - 3 4}$ & 107 & 101 \\
$\mathbf{3 5 - 4 4}$ & 148 & 96 \\
$\mathbf{4 5 - 5 4}$ & 134 & 97 \\
$\mathbf{5 5 - 6 4}$ & 89 & 48 \\
$\mathbf{6 5 +}$ & 30 & 23 \\
\hline
\end{tabular}

Segundo Chávez, Campana e Hass (2003), através de dados divulgados divulgados pelo SINAN e SIM, a faixa etária em que teve mais casos da doença foi acima dos 30 anos, com $55,4 \%$ dos casos. No período de 2001 a 2011 , houve predomínio de pacientes dos 20 aos 59 anos, com aproximadamente $86,5 \%$ dos casos a partir de dados obtidos pela SINAN/MG de acordo com Pudelco, Koehler e Bisetto (2014). Esses dois trabalhos vão de encontro com os resultados deste trabalho.

A maioria das pessoas contaminadas não soube dizer qual a fonte de contaminação, tanto no sexo feminino (342 casos), quanto no masculino (434 casos). O maior número de casos em que se sabia a fonte de contaminação, nos dois sexos, foi o contágio sexual, com 69 casos nos homens e 42 nas mulheres. Diferente desse estudo, outro feito em três centros regionais do estado do Pará, revela que as principais fontes de contaminação na região foram medicamentos injetáveis, tratamento cirúrgico e tratamento dentário (GONÇALVES, 2019).

Já esse estudo coincidiu com este, os quais revelam que a forma de contaminação prevalente foi a mesma, sexual, entretanto, quanto a segunda fonte de contaminação houve divergência, no estudo feito por Pudelco, Koehler e Bisetto foi a vertical e nesse foi a domiciliar. 
Tabela 2. Casos notificados por sexo e mecanismo de infecção da hepatite B.

\begin{tabular}{ccc}
\hline Mecanismo de infecção & $\begin{array}{c}\text { Casos notificados no sexo } \\
\text { masculino }\end{array}$ & $\begin{array}{c}\text { Casos notificados no sexo } \\
\text { feminino }\end{array}$ \\
\hline Ig/Branco & 434 & 342 \\
Sexual & 69 & 42 \\
Transfusional & 3 & 3 \\
Uso de Drogas & 1 & 4 \\
Vertical & 4 & 2 \\
Domiciliar & 18 & 18 \\
Tratamento Cirúrgico & 2 & 1 \\
Tratamento Dentário & 7 & 1 \\
Pessoa/pessoa & 11 & 6 \\
Outros & 4 & 3 \\
\hline
\end{tabular}

[...] A forma de transmissão mais frequente é a sexual, seguida pela vertical que pode ocorrer por via transplacentária, no momento do parto, no aleitamento materno e nos cuidados com o recém nascido (PUDELCO, KOEHLER E BISETTO, 2014, p. 78-86, apud CONCEIÇÃO et al, 2009, p. 5761). Também pode ser transmitido via parenteral, por meio de transfusão sanguínea, reutilização de seringas e agulhas não esterilizadas, colocação de piercing, tatuagens, procedimentos médicos e odontológicos invasivos, acidentes com perfurocortantes e compartilhamento de materiais de higiene (escova dental, depiladores e lamina de barbear). (PUDELCO, KOEHLER E BISETTO, 2014, p. 78-86, apud ATTILIO, 2011, p. 101-6).

Os casos de hepatite B costumam ser notificados na forma clínica crônica, totalizando 945 casos no período estudado (homens: 538 casos e mulheres: 407 casos). A forma clínica aguda possui apenas 12 casos notificados em homens e 4 em mulheres. Há alguns casos em que o diagnóstico foi inconclusivo, o campo na ficha de notificação foi ignorado ou foi hepatite fulminante. 2014):

De acordo com Pudelco (2014, apud GASPAROTO, THOMAZINI e GOLDONI,

No Paraná, verificou-se que a cobertura vacinal entre 1995 a 1999 era abaixo da preconizada pelo Programa Nacional de Imunização do Ministério da Saúde, que é de 95\%. A partir de 1999 essa taxa se eleva e mantém-se até 2011. O estado mantém altas coberturas vacinais em menores de um ano de idade $(97,6 \%)$. A cobertura entre 2001 a 2011 se apresentou mais baixa nas faixas etárias entre 15 a 19 anos (80,5\%) e 20 a 29 anos (82,4\%) (Figura 3). A faixa etária de 10 a 14 anos apresenta alta cobertura vacinal, mas ainda existem muitas crianças que não foram vacinadas no início da implantação da vacina no estado.

Quanto à situação vacinal da hepatite $\mathrm{B}$, na maioria das fichas de notificação esse campo estava em branco, sendo 218 fichas do sexo masculino e 153 do sexo feminino. No sexo masculino, entre os que responderam, 61 tinha a vacina completa, 28 a vacina estava incompleta e 246 não eram vacinados. Já no sexo feminino, 75 pessoas possuíam a vacina completa, 39 a vacina estava incompleta e 155 não eram vacinados. 
Tabela 3. Dados notificados por sexo e faixa etária da hepatite C.

\begin{tabular}{ccc}
\hline Faixa etária & $\begin{array}{c}\text { Casos notificados no sexo } \\
\text { masculino }\end{array}$ & $\begin{array}{c}\text { Casos notificados no sexo } \\
\text { feminino }\end{array}$ \\
\hline$<1$ & 0 & 0 \\
$5-14$ & 0 & 0 \\
$\mathbf{1 5 - 2 4}$ & 20 & 32 \\
$\mathbf{2 5 - 3 4}$ & 25 & 31 \\
$\mathbf{3 5 - 4 4}$ & 60 & 41 \\
$\mathbf{4 5 - 5 4}$ & 66 & 36 \\
$\mathbf{5 5 - 6 4}$ & 38 & 31 \\
$\mathbf{6 5 +}$ & 19 & 13 \\
\hline
\end{tabular}

Esse estudo divergiu deste, pois de acordo com os dados obtidos na região de Cascavel, a cobertura vacinal é pequena. Quanto à hepatite C, a maioria das notificações no sexo masculino foi na faixa etária dos 45 aos 54 anos, com 66 casos. Já no sexo feminino, predominou com 41 casos a faixa etária dos 35 aos 44 anos Então, tanto no sexo masculino, quanto no feminino, o intervalo de idades em que houve maior número de notificações foi dos 35 aos 54 anos. Esse achado é consistente com o estudo de Alvez (2014, tradução nossa) em que houve predomínio de casos de hepatite $C$ na faixa etária entre 40 e 59 anos (60,7\%). Em outro estudo, a partir de dados da Secretaria de Vigilância Epidemiológica do Estado de Santa Catarina, Chávez, Campana e Hass (2003) houve predomínio de casos dos 20 aos 49 anos, o qual teve uma média de idade um pouco mais baixa que este estudo.

Tabela 4. Casos notificados por sexo e mecanismo de infecção da hepatite C.

\begin{tabular}{ccc}
\hline Mecanismo de infecção & $\begin{array}{c}\text { Casos notificados no sexo } \\
\text { masculino }\end{array}$ & $\begin{array}{c}\text { Casos notificados no sexo } \\
\text { feminino }\end{array}$ \\
\hline Ig/Branco & 172 & 146 \\
Sexual & 28 & 17 \\
Transfusional & 7 & 2 \\
Uso de Drogas & 10 & 2 \\
Vertical & 2 & 2 \\
Domiciliar & 2 & 2 \\
Tratamento Cirúrgico & 1 & 1 \\
Tratamento Dentário & 3 & 4 \\
Pessoa/pessoa & 2 & 4 \\
Outros & 1 & 4 \\
\hline
\end{tabular}

Com relação às fontes de contaminação da hepatite $C$, a maioria dos homens (172 casos) e das mulheres (146 casos) não souberam informar quais foram. Entre os que informaram, no sexo masculino predominou a sexual com 28 casos. No sexo feminino também predominou a fonte sexual com 17 notificações. Indo de encontro a isso, "a maior prevalência de infecção pelo HCV tem sido encontrada entre pacientes 
atendidos em clínicas especializadas em doenças sexualmente transmissíveis, entre prostitutas e seus parceiros e entre pacientes coinfectados HIV-HCV" (TEDDER, 1991, p.1299-302, apud MARTINS, SCHIAVON, SCHIAVON, 2010, p.111). Em outro estudo, os maiores fatores de risco foram medicamentos injetáveis, tratamento cirúrgico e tratamento dentário (GONÇALVES, 2019), divergindo dos resultados deste trabalho.

Como na hepatite $B$, os casos notificados da hepatite $C$ costumam ser notificados na forma clínica crônica, sendo 150 casos no sexo masculino e 88 no sexo feminino. A hepatite aguda foi notificada em apenas 3 casos ( 2 homens e 1 mulher). Esse campo foi ignorado na ficha de notificação de 76 casos masculinos e 93 femininos e 2 casos femininos foram inconclusivos.

\section{CONSIDERAÇÕES FINAIS}

Esse trabalho se prontificou a levantar dados sobre a hepetite $B$ e hepatite $C$ nos casos notificados do município de Cascavel/PR para que futuras medidas possam ser tomadas a partir dos resultados obtidos. A partir dos dados coletados observou-se que a hepatite $B$ é mais comum que a hepatite $C$, sendo que teve mais que o dobro de casos notificados no período estudado. Foi possível identificar que na hepatite $\mathrm{B}$ e $\mathrm{C}$, houve predomínio de casos no sexo masculino. Quanto a faixa etária na hepatite $\mathrm{B}, \mathrm{a}$ mais acometida no sexo masculino foi dos 35 aos 44 anos, já no feminino foi dos 25 aos 34. Já na hepatite $C$, predominou a faixa etária dos 45 aos 54 anos no sexo masculino e dos 35 aos 44 anos no feminino, ou seja, pacientes mais velhos que os acometidos pela hepatite B. A faixa etária predominante, em ambos os casos de hepatite, foi a população sexualmente ativa, indo de encontro com a principal fonte de contaminação do estudo: sexual.

Nas fichas de notificação o campo do mecanismo de infecção foi ignorado em mais da metade dos casos, tanto na hepatite $B$, quanto na $C$, o que dificulta uma futura abordagem para redução dos casos dessas doenças. A forma clínica em que a doença foi diagnosticada foi predominantemente crônica, pois só são notificados pela Secretaria de Saúde os casos que são considerados crônicos, pois os agudos não costumam chegar à secretaria, devido ao diagnóstico não ser feito corretamente. Quanto a vacina da hepatite B, por este estudo não tem como concluir sua eficácia, pois a maioria das fichas de notificação não possuíam a situação vacinal registrada e entre as que possuíam, havia pessoas com o esquema completo da vacina que foram diagnosticadas com a doença. Com isso, conclui-se que deve ter a população sexualmente ativa ( 25 aos 54 anos) como prioridade para prevenção da doença, em ambos os sexos. Deve-se ter melhor controle da fonte de contaminação, podendo assim serem tomadas providências para redução do número de contaminados. É necessário melhor controle da situação vacinal nas fichas de notificação, pois na maior parte delas o campo foi ignorado, dificultando assim dizer a eficácia da vacina. Também deve ter supervisionamento das pessoas que estão fazendo o esquema vacinal para que o façam completo e ter maior cobertura vacinal da população.

\section{REFERÊNCIAS}

ALVES, M. dos R. et al. Perfil epidemiológico dos casos de hepatite C em uma diretoria regional de saúde da Bahia. Revista de Pesquisa: Cuidado e Fundamental, [s. I.], 
v. $6, \quad$ n. $3, \quad$ p. 889-896, 2014. Disponível em: $<$ http://search.ebscohost.com/login.aspx?direct=true \&db=foh\&AN=97158033\&lang=p t-br\&site=ehost-live >. Acesso em: 28 jun. 2019

BORTOLUCCI, WC; Ferreira, FN; Correa, NAB. Prevalência de hepatite B no estado do Paraná, Brasil, nos anos de 2008 a 2013. Revista UNINGÁ. 2015; vol 44: 10-16. Disponível em: http://revista.uninga.br/index.php/uninga/article/view/1226/848. Acesso em: 28 jun. 2019.

BRASIL, Ministério da Saúde, Secretaria de Vigilância em Saúde, Departamento de Vigilância Epidemiológica. Hepatites Virais: o Brasil está atento. 2008; 3aㅡ edição. Disponível http://bvsms.saude.gov.br/bvs/publicacoes/hepatites_virais_brasil_atento_3ed.pdf. Acesso em: 20/10/2018.

CHÁVEZ, Juliana Helena; CAMPANA, Sabrina Gonçalves; HAAS, Patrícia. Panorama da hepatite B no Brasil e no Estado de Santa Catarina. Revista Panamericana de Salud Pública, [s.l.], v. 14, n. 2, p.91-96, ago. 2003. Disponível em: $<$ https://www.scielosp.org/scielo.php?script=sci_arttext\&pid=S1020-

$49892003000700003 \&$ Ing $=p t \& n r m=i s o \& t \mid n g=p t>$. Acesso em: 23 jun. 2019. http://dx.doi.org/10.1590/s1020-49892003000700003

CHÁVEZ, Priscila; KOEHLER, Andreia Eloize; BISETTO, Lúcia Helena Linheira. Impacto da vacinação na redução da hepatite B no Paraná. Rev. Gaúcha Enferm., Porto Alegre, v. 35, n. 1, p. 78-86, Mar. 2014 . Disponível em: $<$ http://www.scielo.br/scielo.php?script=sci_arttext\&pid=S1983-

14472014000100078\&Ing=en\&nrm=iso >. Acesso em: 27 jun. 2019. http://dx.doi.org/10.1590/1983-1447.2014.01.37821

GASPAROTO, M. T.; THOMAZINI, C. M.; GOLDONI, A. L. Correlação Entre Cobertura Vacinal E Incidência De Hepatite B Na Região Sul Do Brasil. UNINGÁ Review, [s. I.], v. 20, n. 3, p. 77-81, 2014. Disponível em: <http://search.ebscohost.com/login.aspx?direct=true\&db=foh\&AN=101878565\&lang= pt-br\&site=ehost-live $>$. Acesso em: 27 jun. 2019.

GONÇALVES, Nelson Veiga et al. Hepatites B e C nas áreas de três Centros Regionais de Saúde do Estado do Pará, Brasil: uma análise espacial, epidemiológica e socioeconômica. Cadernos Saúde Coletiva, [s.l.], v. 27, n. 1, p.1-10, 21 fev. 2019. Disponível em: <http://www.scielo.br/scielo.php?script=sci_arttext\&pid=S1414462X2019000100001\&lng=pt\&tlng=pt>. Acesso em: $\overline{28}$ jun. 2019. http://dx.doi.org/10.1590/1414-462x201900010394

MARTINS, T.; NARCISO-SCHIAVON, J. L.; SCHIAVON, L. de L. Epidemiologia da Infecção Pelo Vírus da Hepatite C. Revista da Associação Médica Brasileira, [s. 1.], v. 57 n. 1, p. 107-112, 2011. Disponível em: http://www.scielo.br/pdf/ramb/v57n1/v57n1a24.pdf. Acesso em: 12 jul. 2019.

PUDELCO, Priscila; KOEHLER, Andreia Eloize; BISETTO, Lúcia Helena Linheira. Impacto da vacinação na redução da hepatite B no Paraná. Rev. Gaúcha Enferm., 
Porto Alegre, v. 35, n. 1, p. 78-86, Mar. 2014. Disponível em: $<$ http://www.scielo.br/scielo.php?script=sci_arttext\&pid=S198314472014000100078\&lng=en\&nrm=iso>. Acesso em: 27 Jun. 2019. http://dx.doi.org/10.1590/1983-1447.2014.01.37821

VIEIRA, Gabriel de Deus et al . HEPATITIS B IN RONDÔNIA (WESTERN AMAZON REGION, BRAZIL): descriptive analysis and spatial distribution. Arq. Gastroenterol., São Paulo, v. 52, n. 1, p. 18-21, Mar. 2015. Disponível em: $<$ http://www.scielo.br/scielo.php?script=sci_arttext\&pid=S0004-

28032015000100005\&lng=en\&nrm=iso >. Acesso em: 28 jul. 2019. http://dx.doi.org/10.1590/S0004-28032015000100005

XIMENES, Ricardo Arraes de Alencar et al . Methodology of a nationwide crosssectional survey of prevalence and epidemiological patterns of hepatitis $A, B$ and $C$ infection in Brazil. Cad. Saúde Pública, Rio de Janeiro , v. 26, n. 9, p. 1693-1704, Sept. 2010 Disponível em: <http://www.scielo.br/scielo.php?script=sci_arttext\&pid=S0102-

311X2010000900003\&lng=en\&nrm=iso>. Acesso em: 08 jul. 2019. http://dx.doi.org/10.1590/S0102-311X2010000900003. 